\title{
Selenium Nanoparticles Induce Potent Protective Immune Responses against Vibrio cholerae WC Vaccine in a Mouse Model
}

\author{
Zahra Raahati $\mathbb{D}$, Bita Bakhshi $\mathbb{D}$, and Shahin Najar-peerayeh $\mathbb{C}$ \\ Department of Bacteriology, Faculty of Medical Sciences, Tarbiat Modares University, Tehran, Iran \\ Correspondence should be addressed to Bita Bakhshi; b.bakhshi@modares.ac.ir
}

Received 12 August 2020; Revised 29 November 2020; Accepted 15 December 2020; Published 30 December 2020

Academic Editor: Eirini Rigopoulou

Copyright @ 2020 Zahra Raahati et al. This is an open access article distributed under the Creative Commons Attribution License, which permits unrestricted use, distribution, and reproduction in any medium, provided the original work is properly cited.

\begin{abstract}
The aim of this study was to evaluate the efficacy of selenium nanoparticle (an immune booster) and naloxone (an opioid receptor antagonist) as a new adjuvant in increasing immune responses against killed whole-cell Vibrio cholerae in a mouse cholera model. The Se NPs were synthesized and characterized by UV-visible, DLS, and zeta potential analysis. The SEM image confirmed the uniformity of spherical morphology of nanoparticle shape with $34 \mathrm{~nm}$ in size. The concentration of the Se NPs was calculated as $0.654 \mu \mathrm{g} / \mathrm{ml}$ in the ICP method. The cytotoxic activity of Se NPs on Caco-2 cells was assessed by the MTT assay and revealed $82.05 \%$ viability of cells after $24 \mathrm{~h}$ exposure with $100 \mu \mathrm{g} / \mathrm{ml}$ of Se NPs. Female BALB/C mice were orally immunized three times on days 0,14 , and 28, and challenge experiments were performed on immunized neonates with toxigenic $V$. cholerae. Administration of Se NP diet led to significant increase in $V$. cholerae-specific IgG and IgA responses in serum and saliva and caused protective immunity and $83.3 \%$ survival in challenge experiment against 1 LD50 V. cholerae in a group receiving diet of Se NPs compared with other groups including Dukoral vaccine. The IL-4 and IL-5 were significantly increased in response to WC+daily diet of Se NPs with or without naloxone. Naloxone proved no effect on IL-4 and IL-5 increase and is proposed as null in the cytokine and antibody production process. These results reveal that daily diet of Se NPs could efficiently induce immune cell effectors in both humoral and mucosal levels.
\end{abstract}

\section{Introduction}

Cholera disease is an acute diarrhea with an old history caused by Vibrio cholerae, a gram-negative and motile bacterium. Cholera toxin (CT) is responsible for the watery diarrhea which is produced by toxigenic Vibrio cholerae [1]. Since the year 1817, there have been 7 pandemics of cholera in the world. Cholera pandemic was caused by $V$. cholerae biotypes $\mathrm{O} 1$ and 0139 [1]. Today, cholera remains a global problem in many parts of the world, and from 2000 to 2016, 3.4 million cholera cases and 65600 deaths occurred worldwide [2].

Vaccination is a complementary strategy for prevention and control of cholera diseases alongside providing sanitation and safe water. Mucosal surfaces are considered the first line of host-microbe interactions that cause the establishment and maturation of mucosa-associated lymphoid tissues and affect the induction and initiation of innate together with acquired mucosal immune responses. Mucosal vaccination has some functional and practical advantages, including inferior costs and no pain and risk of needle-stick injuries and subsequent blood-borne diseases. Moreover, mucosal vaccination can also induce both humoral- and cell-mediated immune responses in both systemic and mucosal compartments and can efficiently induce long-lasting $B$ cell- and T cell-associated memory [3, 4]. Accordingly, oral delivery can induce production of antigen-specific sIgA in the gastrointestinal tract, salivary glands, and mammary glands [3]. Several antigens of $V$. cholerae have been studied as inducers of the immune system against bacteria, but these antigens create only a short-term protection if applied alone [5]. Currently, two types of oral killed cholera vaccine are available and have global license: killed whole-cell (WC) Vibrio cholerae O1 plus recombinant CTB (Dukoral) and killed whole-cell bivalent $\mathrm{O} 1$ and $\mathrm{O} 139$ (Shancol). Oral cholera vaccines have been instrumental in controlling disease in endemic areas, but the protective immunity initiator by these vaccines is low-level and short-term [6]. However, in Russia, 
an oral vaccine against cholera diseases, other than the global commercial cholera vaccines, is being used which is a combination of cholera toxoid and $\mathrm{O}$-antigens that has been shown to induce a high protective immune response and is stable in human for 6 months [7].

With the development of nanoscience, recent studies on vaccines have led to the use of nanoparticle in vaccine formulations or as adjuvants alongside vaccine components. Selenium element is one of the rare and essential minerals in the body with various functions such as anticancer and antiviral and is part of the antioxidant enzymes [8-11]. Selenium in the nanoscale has attracted considerable attentions in the present era due to the high bioavailability and low toxicity. Selenium nanoparticles (Se NPs) in the laboratory are made chemically or biogenically and have been proven functional in stimulating the immune system through various pathways, such as innate immunity, T cells, and NK cells [12].

Furthermore, opioids are in fact a collection of opiate-like substances. Naloxone (Nal) is an opioid antagonist, previously studied as an effector to shift immune response to cellular immunity by Th1 response $[13,14]$.

The aim of this study was to examine the adjuvant activity of naloxone and Se NP and increase immune responses and protective immunity against Vibrio cholerae whole cells in challenge experiment of cholera in a mouse model.

\section{Materials and Methods}

2.1. Ethical Consideration. The study was evaluated and approved by the research ethics committee of Tarbiat Modares University under IR.MODARES.REC.1397.068 approval ID before it began. All methods were carried out in accordance with relevant guidelines and regulations in manuscript which have been reviewed by the animal care committee of Tarbiat Modares University. Personnel involved in this research were trained and successfully passed the animal care courses.

2.2. Bacterial Strains. V. cholerae ATCC 14035, a classical O1 serotype, was used for oral immunization and challenge experiments. Approximately $10^{8} \mathrm{CFU}$ (colony-forming unit) of killed whole-cell $V$. cholerae was used for mouse immunization. $V$. cholerae ATCC 14035 in a 50\% lethal dose was used in neonatal mouse challenge.

2.3. Inactivation of $V$. cholerae. $V$. cholerae cells were cultured overnight in $\mathrm{BHI}$ agar at $37^{\circ} \mathrm{C}$, and a fresh 18 -hour subculture was prepared in LB broth. Bacterial cells were washed three times with PBS and diluted to $10^{9}$ cells $/ \mathrm{ml}$ and killed with $1 \%$ formaldehyde-PBS for $2 \mathrm{~h}$. Cells were washed twice with $\mathrm{PBS}$ and stored at $4^{\circ} \mathrm{C}$ for future use [15].

2.4. Synthesis of Selenium Nanoparticles (Se NPs). Se NPs were synthesized by the reduction of sodium selenite by the ascorbic acid. Ascorbic acid (Merck, Germany) in a concentration of $58.13 \mathrm{mM}$ was slowly added to $1.2 \mathrm{mM}$ $\mathrm{Na}_{2} \mathrm{SeO}_{3}\left(5 \mathrm{H}_{2} \mathrm{O}\right)$ (Merck, Germany) under a stirrer at $1300 \mathrm{rpm}$. The solution was washed twice and resuspended in $1 \mathrm{ml}$ deionized water. Tween $20(30 \mu \mathrm{l} / 20 \mathrm{ml})$ was used to prevent the aggregation of selenium nanoparticles during the synthesis procedure [16].

2.5. Characterization of Se Nanoparticles. The identity of Se NPs was confirmed by UV-vis absorption spectroscopy (PerkinElmer, Lambda 25) at 200-500 nm. The size distribution of Se nanoparticles and the surface charge of the nanoparticles (zeta potential) were determined by the dynamic light scattering (DLS) analysis using Malvern Zetasizer Nano-ZS instrument.

The morphology and particular size of Se NPs were determined by scanning electron microscopy (SEM, FEI Company of USA, model Quanta 200). Nanoparticles in MilliQ water were allowed to slowly dry on a glass slide and covered with a layer of gold metal before picture documentation.

Selenium content of the nanoparticles was determined by Inductively Coupled Plasma-Atomic Absorption Spectroscopy (ICP-AAS). Acid digestion of nanoparticles was carried out using a solution of $2 \%$ nitric acid. Selenium standards were then prepared from sodium selenite salt at concentrations of 1-100 ppm, and the concentration of nanoparticles was measured based on the standard concentration of selenium.

2.6. Cell Culture and Assessment of Cytotoxicity of Se NPs. The toxicity of Se NPs was calculated by the MTT assay. Caco-2 cell lines were purchased from Iranian Biological Resource Center. The Caco- 2 cells were cultured in DMEM containing fetal bovine serum (10\%) and penicillin and streptomycin at $37^{\circ} \mathrm{C}$ at $5 \% \mathrm{CO}_{2}$. Cells were centrifuged for $5 \mathrm{~min}$ at $1500 \mathrm{rpm}$ and counted on the Neubauer chamber with $1 / 2$ trypan blue, and the appropriate volume of cell suspension $\left(10^{5}\right.$ cells) was seeded to a 96 -well plate [17]. The cells were incubated with $50-200 \mu \mathrm{g} / \mathrm{ml}$ Se NPs for 24 and $48 \mathrm{~h}$ after which $100 \mu \mathrm{l}$ DMEM medium containing reconstituted MTT $(10 \mathrm{~mL})$ was added and the plates were returned to the incubator. After $4 \mathrm{~h}$, the medium was removed and $100 \mu \mathrm{l}$ of detergent reagent (DMSO) was added to solubilize formazan crystals. Optical densities at $570 \mathrm{~nm}$ were read, and the percentage of live cells was calculated relative to the control cells.

2.7. Animals. The immune responses were evaluated in 4-6week-old female BALB/C mice purchased from Pasteur Institute of Iran. The mice were kept in 8 groups in separate cages under normal condition, and each group consisted of 6 mice [18]. The mice were acclimated for 5 days before beginning the experiment, and during this time, the animals were exposed to the standard condition of temperature and humidity. Experiment on animals was carried out in accordance with relevant guidelines and regulations of the Institute of Laboratory Animal Resources [19].

2.8. Mouse Immunization. Animals were kept fasted for $5 \mathrm{~h}$ and subjected to gastric acid neutralization with $5 \%$ sodium bicarbonate. The immunization of mice was performed on days 0,14 , and $28[20,21]$. Mouse groups are summarized in Table 1. 
TABLE 1: Formulation of suspension for each group of mice.

\begin{tabular}{lc}
\hline Group & Suspension received \\
\hline WC+Nal & $250 \mu \mathrm{l}$ of PBS containing inactivated Vibrio cholerae $\left(10^{8} \mathrm{CFU}\right)$ and naloxone $(0.15 \mathrm{mg})$ \\
WC+Se NPs & $250 \mu \mathrm{l}$ of PBS containing inactivated Vibrio cholerae $\left(10^{8} \mathrm{CFU}\right)$ and selenium NPs $(100 \mu \mathrm{g})$ \\
WC+diet of Se NPs & $250 \mu \mathrm{l}$ of PBS of inactivated Vibrio cholerae $\left(10^{8} \mathrm{CFU}\right)$, daily diet of Se NPs $(100 \mu \mathrm{g})$ \\
WC+Nal+Se NPs & $250 \mu \mathrm{l}$ of PBS containing inactivated Vibrio cholerae $\left(10^{8} \mathrm{CFU}\right)$ and naloxone $(0.15 \mathrm{mg})$ and selenium NPs $(100 \mu \mathrm{g})$ \\
WC+Nal+diet of Se & $250 \mu \mathrm{l}$ of PBS containing inactivated Vibrio cholerae $\left(10^{8} \mathrm{CFU}\right)$ and naloxone $(0.15 \mathrm{mg})$, daily diet of Se NPs \\
NPs & $(100 \mu \mathrm{g})$ \\
Dukoral vaccine & $200 \mu \mathrm{l}$ of PBS containing Dukoral vaccine $\left(10^{8} \mathrm{CFU}\right)$ \\
Control & $250 \mu \mathrm{l}$ of inactivated Vibrio cholerae $\left(10^{8} \mathrm{CFU}\right)$ \\
Control & $250 \mu \mathrm{l}$ of PBS \\
\hline
\end{tabular}

WC and Se NPs were used by the intraintestinal route with standard gavage. In the group that diet of selenium was used, mice received a daily dose of $100 \mu \mathrm{g}$ of selenium NPs on days 0 to 42 .

Naloxone has no absorption from the gastrointestinal tract, and according to the instructions, the naloxone drug was injected.

2.9. Sample Collection. Two weeks after each immunization, on days $0,14,28$, and 42 , blood and saliva samples of mouse groups were collected $[20,21]$. Blood samples were incubated at $4^{\circ} \mathrm{C}$ for $60 \mathrm{~min}$ and centrifuged at $10000 \mathrm{rpm}$ for $10 \mathrm{~min}$, and serum was separated. The saliva sample was collected with a sterile swab from the mouth and was stored in $100 \mu \mathrm{l}$ of sterile PBS. Saliva and separated serum were stored at $-20^{\circ} \mathrm{C}$ for further analysis.

2.10. Measurement of Antibody Responses. Enzyme-linked immunosorbent assay (ELISA) was used to measure the total IgG antibodies in serum samples. ELISA plates were coated with $10^{8} \mathrm{CFU}$ of whole-cell $V$. cholerae as an antigen and incubated at $4^{\circ} \mathrm{C}$ overnight. Nonbinding sites were blocked with $2 \% \mathrm{BSA}$ at $37^{\circ} \mathrm{C}$ for $2 \mathrm{~h}$. Plates were washed three times with PBS-0.05\% Tween 20 (PBST), and $100 \mu \mathrm{l}$ of sera in $1 / 100$ through $1 / 12800$ dilutions was added to each well and incubated at $37^{\circ} \mathrm{C}$ for $2 \mathrm{~h}$. After washing steps, $100 \mu \mathrm{l}$ of peroxidase-conjugated goat anti-mouse IgG (Sigma-Aldrich) diluted in PBST $(1: 10000)$ was added to each well. Plates were incubated for additional $1.5 \mathrm{~h}$ at $37^{\circ} \mathrm{C}$. After washing 3 times, $100 \mu \mathrm{l}$ of tetramethylbenzidine (TMB) was added to wells and incubated in the darkness for $30 \mathrm{~min}$. The reaction was stopped by $1 \mathrm{M} \mathrm{H}_{2} \mathrm{SO}_{4}$, and the absorbance values were read by using an ELISA reader (Labsystems, model no. 352) at $450 \mathrm{~nm}$ [22]. An IgA antibody in saliva and serum samples was evaluated in the same procedure with goat anti-mouse IgA in 1/10000 dilution (SIGMA Aldrich) as the secondary antibody. Serum and saliva dilutions for IgA antibody evaluation was $1 / 100$ through $1 / 12800$ dilutions for serum and 1/25-1/800 dilutions for saliva.

2.11. Cytokine Assays. Cytokine production (IL-4, IL-5) was assessed on blood samples by the ELISA method using the R\&D Systems kit. Briefly, $100 \mu$ l capture antibody was added to each well of plate and was incubated at room temperature overnight. Plates were washed three times with wash buffer (PBS-0.05\% Tween 20). Then, Reagent Diluent was added to each well and was incubated for $1 \mathrm{~h}$. Following washing, $100 \mu \mathrm{l}$ serum was added to wells and was incubated for $2 \mathrm{~h}$. After washing, a detection antibody was added and was incubated for $2 \mathrm{~h}$ and then was washed again. Streptavidin-HRP was added and was incubated for $20 \mathrm{~min}$. $100 \mu \mathrm{l}$ TMB was added and was incubated in a dark place for $20 \mathrm{~min}$. Stopper solution $\left(2 \mathrm{~N} \cdot \mathrm{H}_{2} \mathrm{SO}_{4}\right)$ was added, and the absorbance was measured with an ELISA plate reader at $450 \mathrm{~nm}$.

2.12. Challenge Experiments. To assess protective immunity, challenge experiments were performed on 3- to 5-day-old unimmunized pups. The experiment included 8 groups, and each consisted of 6 pups. The neonates were kept separated from dams at $30^{\circ} \mathrm{C}$, and their standard condition of temperature and humidity was controlled every $4 \mathrm{~h}$ for $48 \mathrm{~h}$. Death and health of mice were recorded every $4 \mathrm{~h}$. A total of $50 \mu \mathrm{l}$, containing $25 \mu \mathrm{l}$ of immune serum from immunized mice on day 42 plus $25 \mu \mathrm{l}$ of LB broth containing $10^{6} \mathrm{CFU}$ of $V$. cholerae ATCC 14035 , was given to pups orally $[22,23]$. The measurement criteria of the challenge were evaluation of animals' resistance in the control group compared with animals injected with immune serum followed by evaluation of elapsed time of death of resistant animals compared with the control group.

2.13. Statistical Analyses. The ANOVA test and $t$-tests were used to assess the significant differences. In neonate challenges, we analyzed survival curves by the log-rank test. The significant values were considered less than 0.05. All antibody responses and cytokine measurements were performed in triplicate, and the results were presented as the mean of experiments \pm standard deviation.

\section{Results}

3.1. Characterization of Se NPs. The UV-visible absorption spectra of nanoparticle showed a maximum absorption value at around $260 \mathrm{~nm}$ (Figure 1). The appearance of a sharp extinction peak around the specified wavelength with low FWHM (full width at half maximum) confirmed the conversion of selenite ions into Se NPs and monodispersity of the 


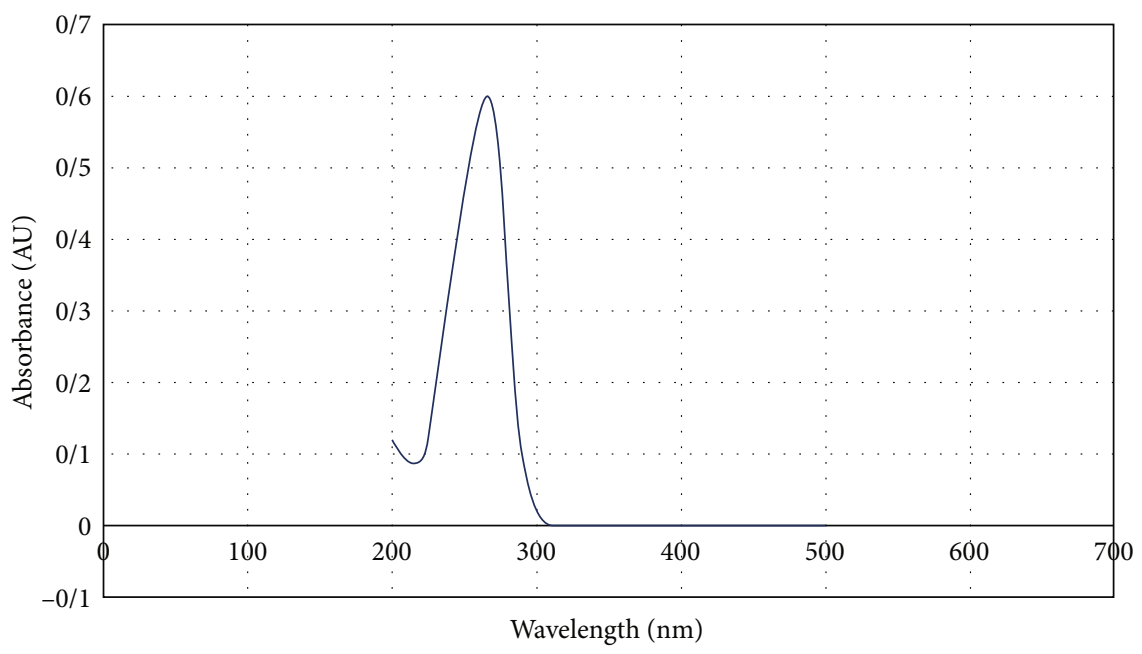

FIGURE 1: UV-vis spectrum of Se NPs.

nanoparticles. The characteristic orange color of Se NP colloidal suspension was also visually evaluated.

According to the result of the DLS analysis, an average hydrodynamic diameter of synthesized Se NPs was $48 \mathrm{~nm}$. The polydispersity index (PDI) in DLS analysis was 0.089 (Figure 2(a)) that confirmed the homogeneity and nondispersion of the nanoparticle size. Zeta potential of the nanoparticle was $-21 \mathrm{mV}$ which was detected by using a zeta sizer (Figure 2(b)).

The result of the SEM image indicated spherical morphology of nanoparticle shape with $34 \mathrm{~nm}$ in size. These results also confirmed the uniformity of the nanoparticles size and morphology (Figure 3).

In the ICP method, the calibration curve of the selenium standards was plotted and the concentration of the Se NPs was calculated as $0.654 \mu \mathrm{g} / \mathrm{ml}$ (Figure 4 ).

3.2. Cytotoxic Effect of Se NPs. The cytotoxic activity of Se nanoparticle on Caco-2 cells was analyzed by the MTT assay. As represented in Figure 5, in cells treated with 50-200 $\mu \mathrm{g} / \mathrm{ml}$ of Se NPs for 24 and $48 \mathrm{~h}$, more than $50 \%$ of the cells were viable. Moreover, the viability of cells in $100 \mu \mathrm{g} / \mathrm{ml}$ of Se NPs (concentration used in this study) after 24 and $48 \mathrm{~h}$ was $82.05 \%$ and $77.04 \%$, respectively.

3.3. Antibody Responses. ELISA was performed on serum and saliva samples 14,28 , and 42 days after immunization. The highest level of the antibody was observed on day 42 . Following oral immunization, we found significant increase in IgG response in all immunized groups compared with the mice that only received PBS $(P<0.05)$. There has been a significant increase in IgG response in groups receiving selenium nanoparticle diet compared with whole-cell control group $(P<0.01)$. Moreover, in the diet groups, the IgG antibody level was significantly higher than that in the Dukoral vaccine group $(P<0.05)$. Moreover, a significant difference was observed in antibody responses of the group immunized with Se NPs-whole-cell group with the same group receiving an Se NP regimen $(P<0.05)$. In the mouse group that were immunized with whole cells in combination with naloxone, no substantial difference was observed in comparison with the whole-cell control group $(P>0.05)$ (Figure 6).

In the case of IgA antibody response, there was a significant antibody response in the serum sample of immune mice compared with the PBS control group $(P<0.05)$. In serum and saliva samples, much higher level of the IgA antibody was observed in groups with a selenium NP regimen (Figures 7(a) and 7(b)). A significant rise was detected in IgA level in the serum sample of mice with selenium diet groups in contrast with Dukoral vaccine and control groups $(P<0.05)$. No significant difference was detected between groups WC-Nal, WC-NP, WC-Nal-NP, and Dukoral compared with whole-cell alone group $(P>0.05)$. In saliva samples, a significant increase in IgA antibody response in the WC-diet of Se NP, WC-Nal-diet of Se NPs, and Dukoral vaccine group was detected in comparison with that in the whole-cell alone group $(P<0.05)$.

3.4. Measurement of Cytokine. Indirect ELISA was performed to investigate the level of IL-4 and IL-5 after immunization. The result indicated that IL-4 was increased in the serum sample of all immune mouse groups. In fact, two groups with selenium diet showed the highest level of IL-4, among which the WC-diet of Se NP group revealed significant increase compared with the Dukoral vaccine $(P<0.05)$ and wholecell control groups $(P<0.01)$. Furthermore, in the immune mouse group, there was no significant disparity of IL-4 level in the whole-cell-Nal and whole-cell-Nal-NP groups in contrast with the whole-cell control group $(P>0.05)$ (Figure 8(a)). There was a significant increase in IL-5 level in mouse groups immunized with WC-Nal-NP diet of Se NPs compared with Dukoral vaccine $(P<0.05)$ and wholecell control groups $(P<0.05)$ (Figure $8(b))$.

3.5. Protection Assay. In neonatal mouse challenges, death was recorded every $4 \mathrm{~h}$ for $48 \mathrm{~h}$, after gavage injection of compounds. No death was recorded within the first 24 hours. According to Figure 9, in the control group (PBS), mice started dying at the $24^{\text {th }}$ hour and all of them died during the $38^{\text {th }}$ hour. Overall, the death of the other groups started 


$\begin{array}{rllll} & & \text { Size (d.nm): } & \text { \% intensity: } & \text { St dev (d.nm): } \\ \text { Z-average (d.nm): } 48.08 & \text { Peak 1: } & 52.44 & 100.0 & 15.51 \\ \text { Pdl: } 0.089 & \text { Peak 2: } & 0.000 & 0.0 & 0.000 \\ \text { Intercept: } 0.960 & \text { Peak 3: } & 0.000 & 0.0 & 0.000 \\ \text { Result quality: Good } & & & & \end{array}$

Size distribution by intensity

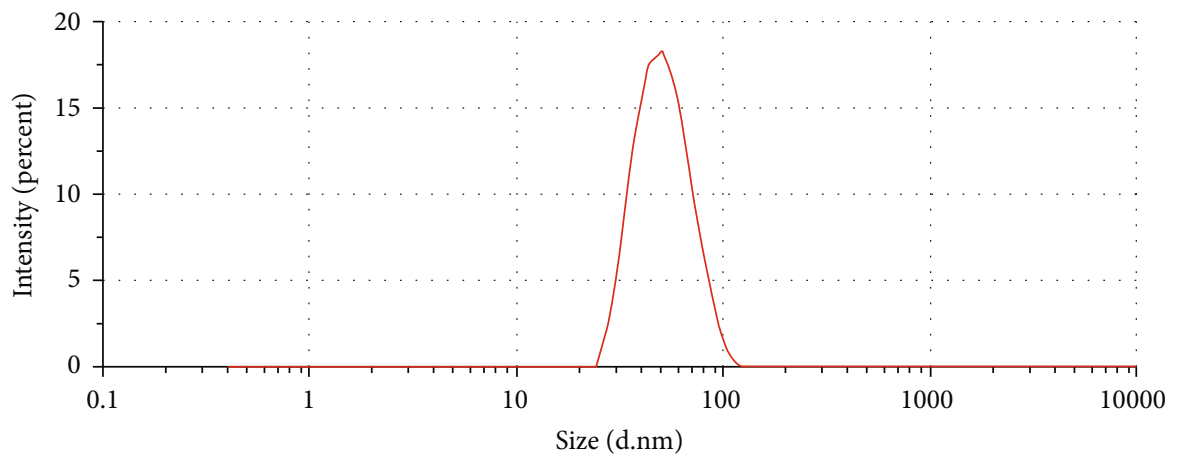

(a)

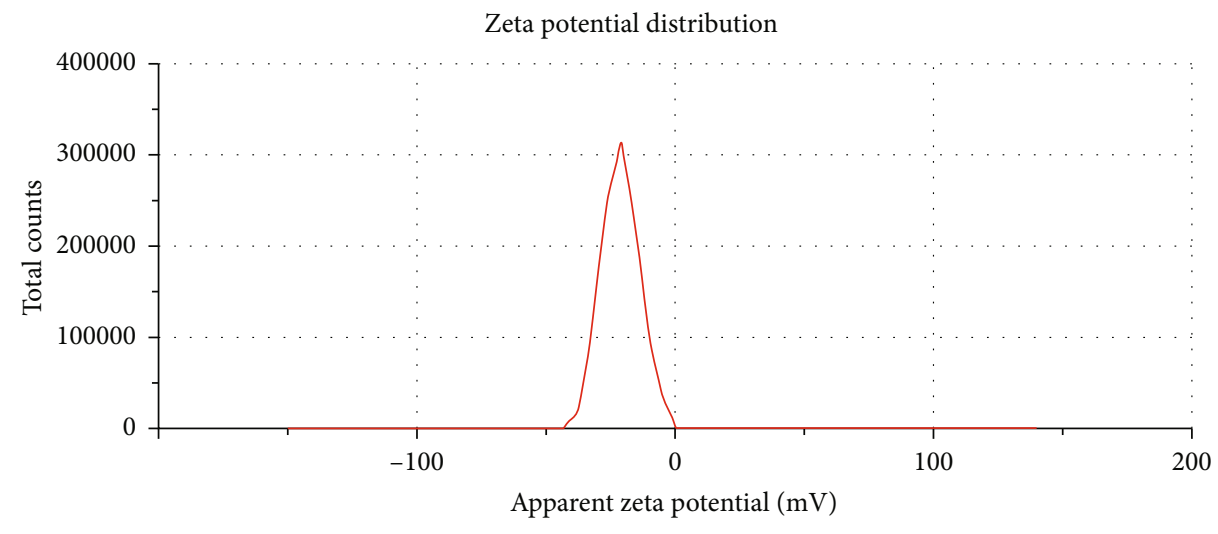

— Record 12: 31

(b)

Figure 2: (a) DLS analysis of synthesis of Se NPs. (b) Zeta potential of synthesis of Se NPs.

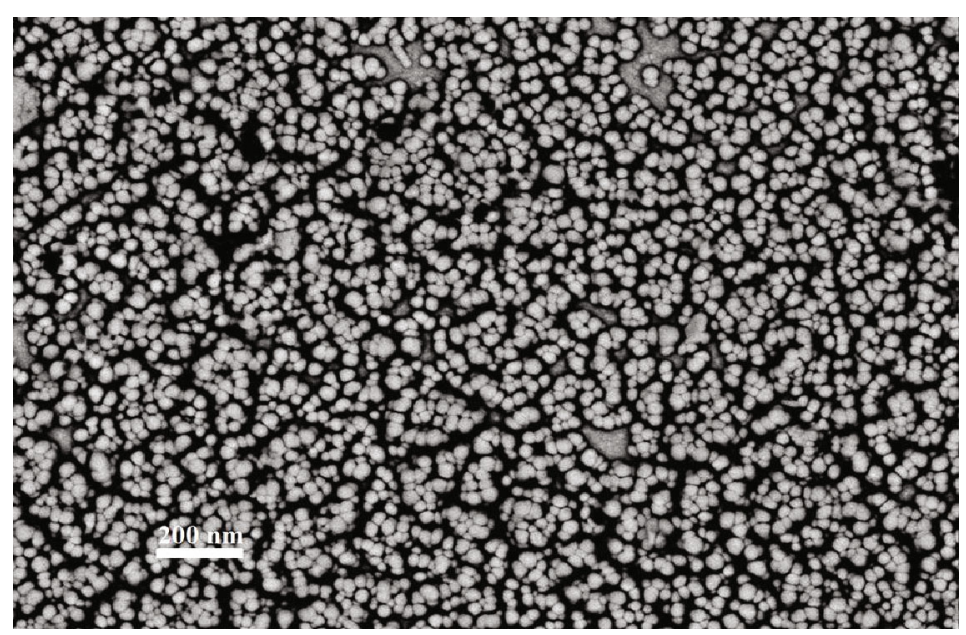

Figure 3: SEM image of synthesis of Se NPs. 


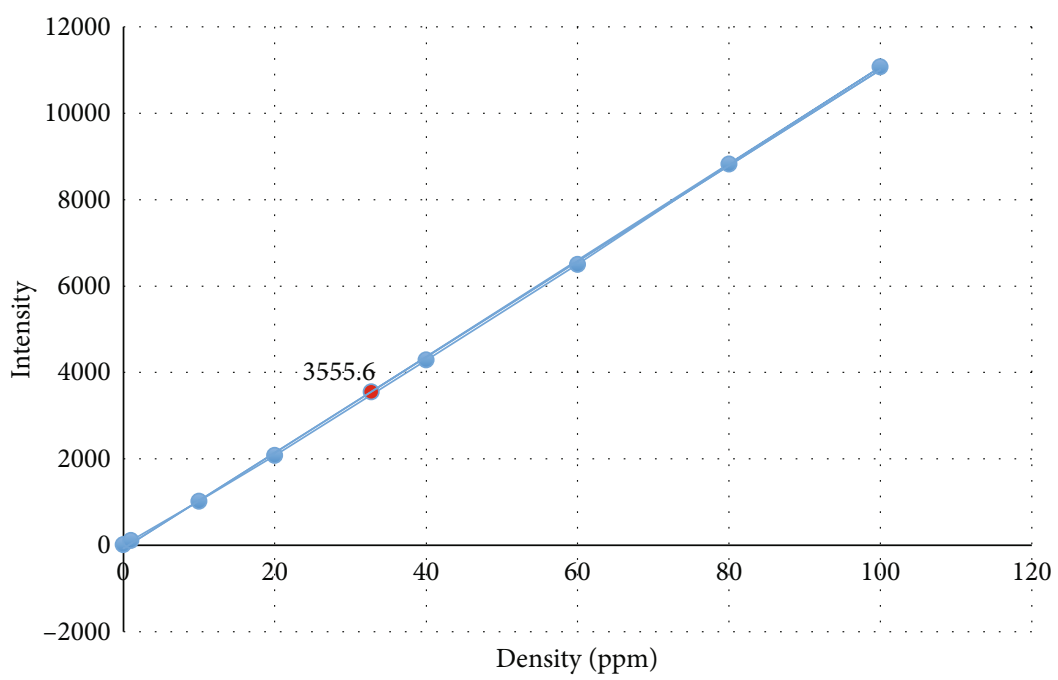

FIGURE 4: Calibration curve of concentration of standard selenium solutions.

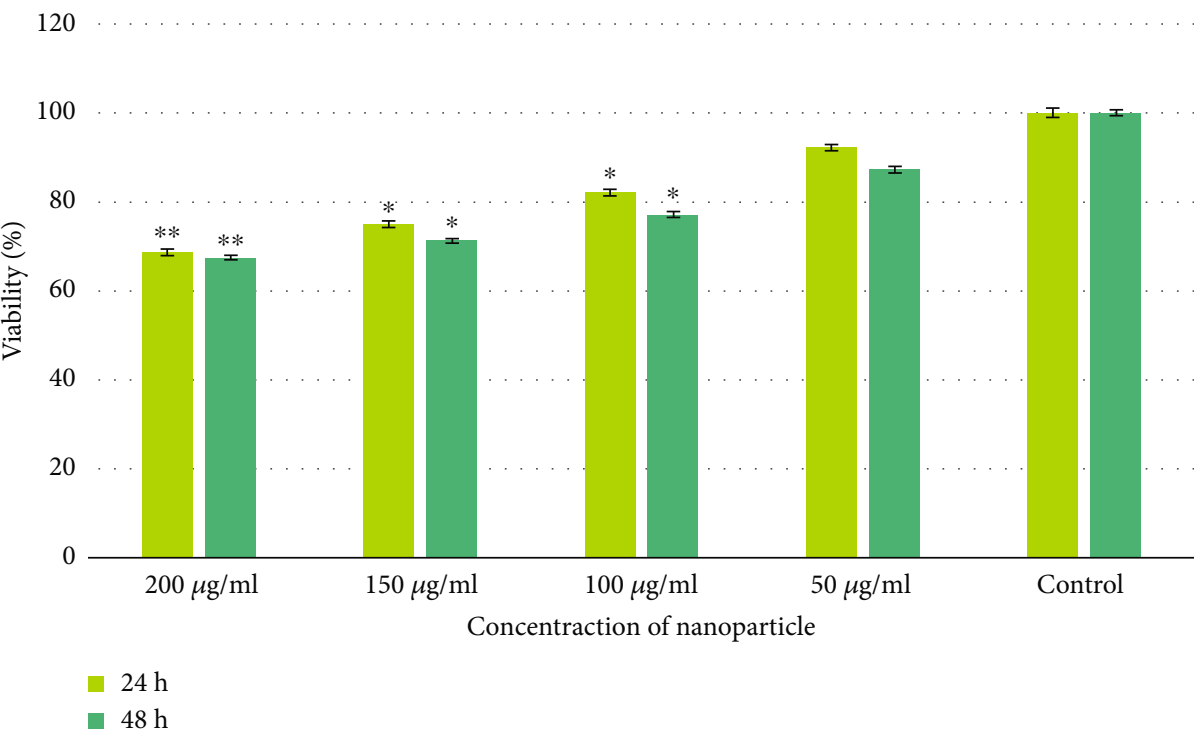

Figure 5: Viability of Caco-2 cells $\left({ }^{*} P<0.05,{ }^{* *} P<0.01\right)$.

at 24,28 , and $38 \mathrm{~h}$ after injection, and all of them were more resistant than the control group.

Significant protection was observed against 1 LD50 V. cholerae in the group receiving diet of Se NPs (83.3\% survival) compared with others. However, the WC-Se NP and WC-Se NP-Nal groups have the same survival rate as the Dukoral group (66.6\% survival). The WC-Nal group like the whole-cell alone group showed a $33.3 \%$ survival rate (Figure 9) $(P<0.001)$.

\section{Discussion}

Two commercially available oral vaccines against $V$. cholerae, Dukoral and Shancol, are associated with killed whole-cell Vibrio cholerae [24]. These oral cholera vaccines have inclusive license, and the World Health Organization uses them globally in the endemic region, in parallel with improvements in sanitation and safe water that lead to cholera control and prevention $[25,26]$. Dukoral is one of the oral cholera vaccines that contain killed Vibrio cholerae $\mathrm{O} 1$ classical and El Tor biotype whole cells together with the recombinant cholera toxin B subunit [27]. The vaccine is applicable in adults and children $>2$ years of age with protective duration of about $50 \%$ over 3 years [28]. Shancol is a variant of whole-cell bivalent vaccine consisting of killed whole cells of $V$. cholerae serogroups $\mathrm{O} 1$ and $\mathrm{O} 139$ that is licensed for administration in adult and children older than 1 year [29, 30]. However, some studies on protective immunity of oral killed vaccine have suggested $79-86 \%$ protection for 6 months [31-33]. In fact, the protective immunity initiator by these vaccines is low-level and short-term [6]. Incorporation of adjuvants along with vaccine ingredients will increase 


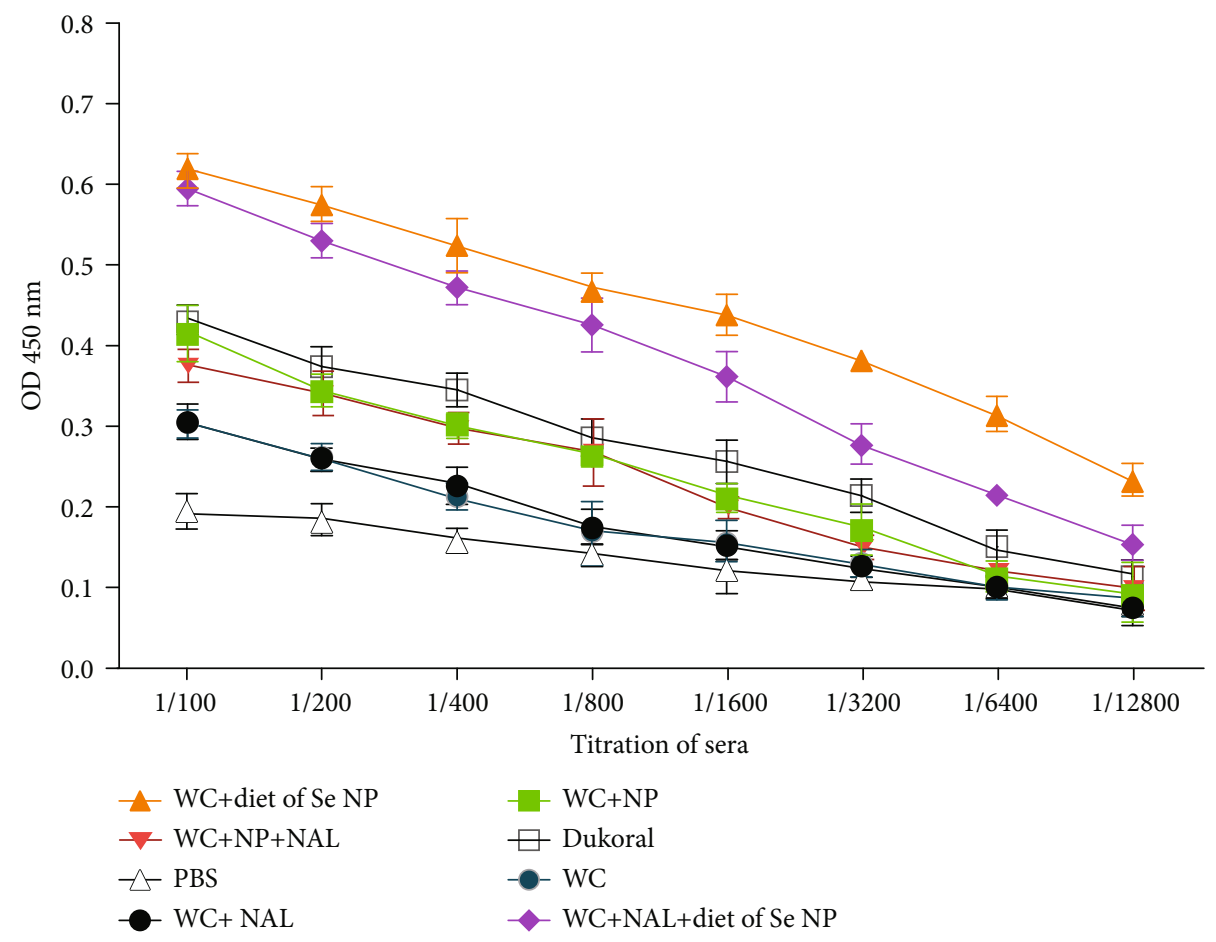

FIGURE 6: Titration of IgG antibody in sera in different immunized mouse groups on day 42.

the effect of the vaccine component and thus improve protective immunity. Dukoral vaccine has used an rCTB antigen as both immunogen and adjuvant, alongside the killed whole cells, and has improved the vaccine efficiency. Several antigens of $V$. cholerae have been studied as inducers of the immune system against bacteria, such as lipopolysaccharide, toxins, and outer membrane vesicles. Studies on cholera toxin reveal that these antigens create only a short-term protection if applied alone [5], and it is better to be used in combination with the killed whole-cell component. Other $V$. cholerae subunit antigens may elicit a good immune response as immunogen, but generally, their production requires lots of cost and a cold chain for storage and transportation. Despite extensive studies on different $V$. cholerae antigens as immunogens and their different efficacy, commercial vaccines against cholera still contain whole cells as the main component [24].

Immunoglobulin $\mathrm{G}$ (IgG) and secretory immunoglobulin A (sIgA) antibody responses to $V$. cholerae antigens play the most important role in protection. IgG antibodies specific for $V$. cholerae antigens are protective against cholera in the mouse model, and specific intestinal secretory IgA induced against $V$. cholerae creates the most protection [34]. Compared with parenteral killed whole-cell vaccines, oral vaccines provide long-term protection, and it is also worth noting that gastrointestinal immunity induces a secretory antibody that plays a key role in preventing bacterial attachment to the intestinal tract [34].

Research on modern vaccines with new ingredients could be useful, and it is proposed that it is better to use adjuvants in conjunction with vaccine compartment to increase the duration of protective immunity of oral vaccines. In a recent research on cholera nanovaccine, for example, gold nanoparticle has been used in conjugates with different Vibrio cholerae protective antigens and evaluated in two animal models (rabbit and mice), which showed high level of antitoxic antibodies and protective immunity compared with a common vaccine (control) [35]. Moreover, Vibrio cholerae lipopolysaccharide-loaded chitosan nanoparticles have been shown to induce a good immune response and high level of IgA, IgG, and IgM, along with a member of bacteria. These studies show that nanoparticles, in the role of an adjuvant or carrier, can be useful in inducing immune response in the cholera vaccine model [36].

In this study, two nonantigenic adjuvants, selenium nanoparticle and naloxone, were applied with killed whole cells, and their role in increasing the immune responses and protective efficacy of vaccine was investigated. The selenium nanoparticles synthesized and applied in this study were of uniform size with no agglomeration and little toxicity in vitro for Caco- 2 cells. When selenium as an adjuvant was used in conjunction with WC, a significant increase was observed in mouse immune responses in comparison to the control group (WC), while in mice immunized with whole cell+daily diet of Se NPs, superior immune responses and protective efficacy were observed. In fact, administration of Se NP diet leads to significant increase in $V$. cholerae-specific $\operatorname{IgG}$ and $\operatorname{IgA}$ responses in serum and saliva and protective immunity in challenge experiment in comparison with control groups.

IL-4 and IL-5 were significantly increased in response to WC+daily diet of Se NPs with or without naloxone, and both of cytokines are supposed to be involved in activation, proliferation, and terminal differentiation of antigen-reactive IgA 


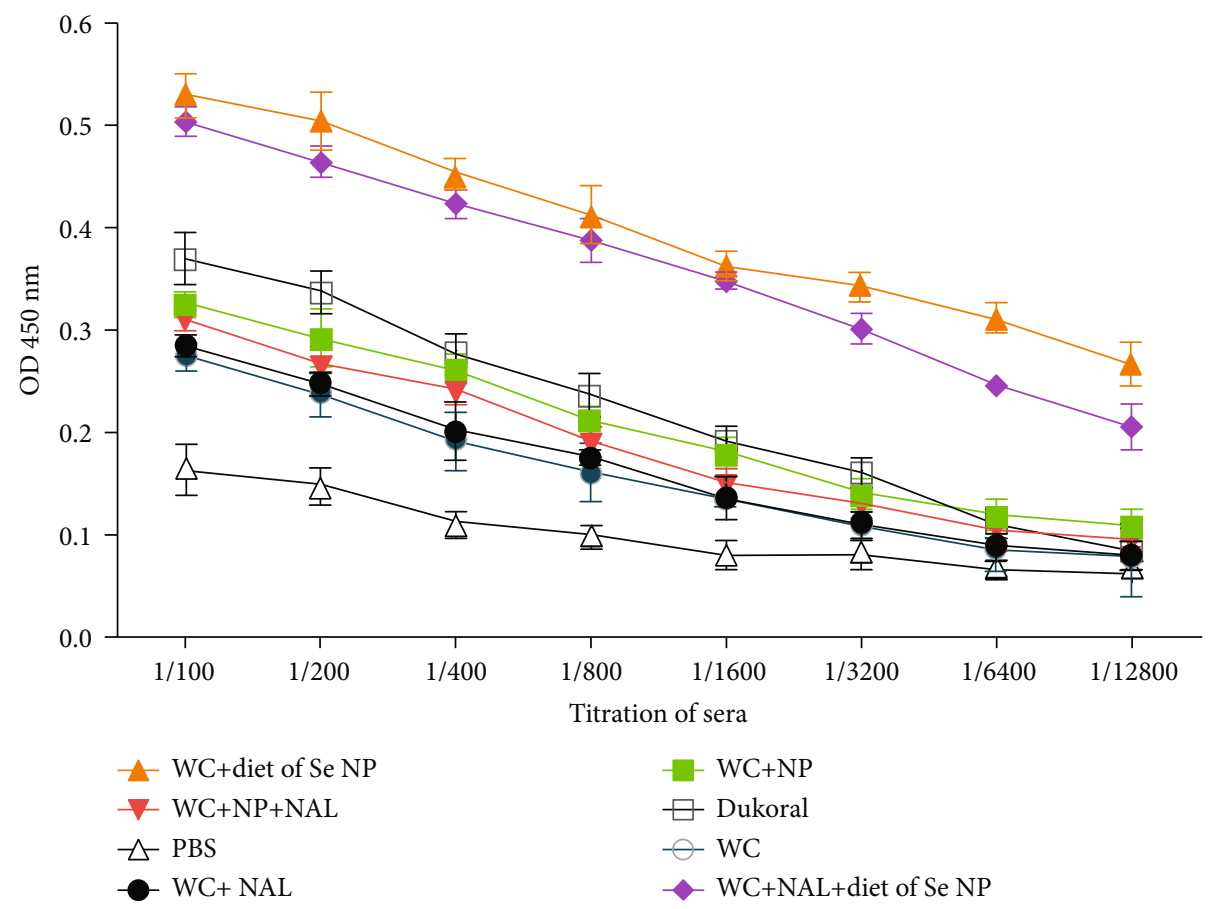

(a)
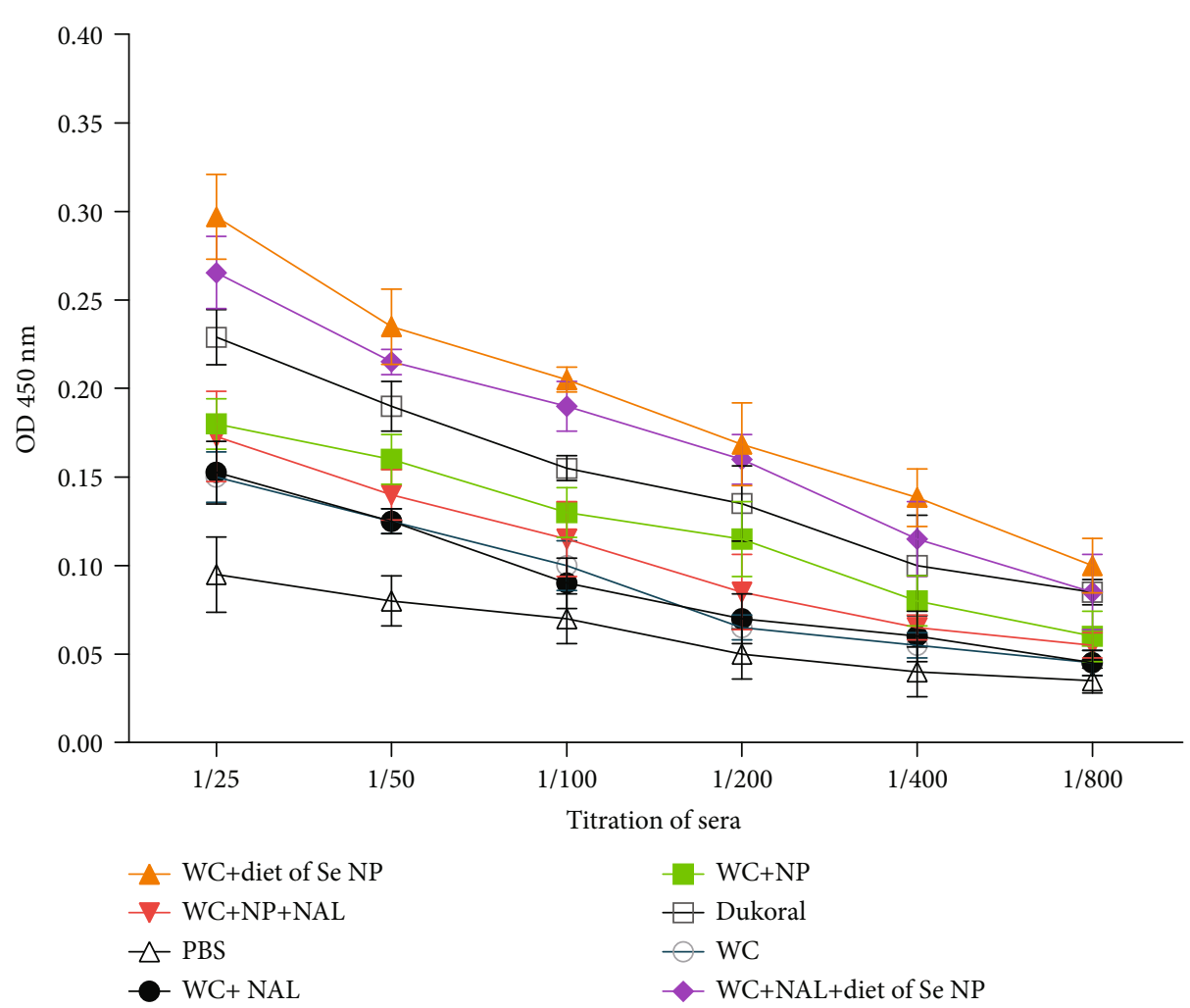

Titration of sera

$$
\begin{aligned}
& \square \text { WC+NP } \\
& \square \text { Dukoral } \\
& - \text { WC } \\
& - \text { WC+NAL+diet of Se NP }
\end{aligned}
$$

(b)

Figure 7: (a) IgA titer in serum of different mice on day 42. (b) IgA titer in saliva of different mice on day 42.

B cells to plasma cells and consequent antibody production. Moreover, IL-5 contributes to isotype switching of B cells to pIgA-producing plasma cells. This reveals that daily diet of Se NPs could efficiently induce immune cell effectors in both humoral and mucosal levels and increased immune responses due to daily diet of selenium NPs were even higher than those due to the commercial Dukoral vaccine; however, naloxone proved no effect on IL-4 and IL-5 increase and is proposed as null in the cytokine and antibody production process. 


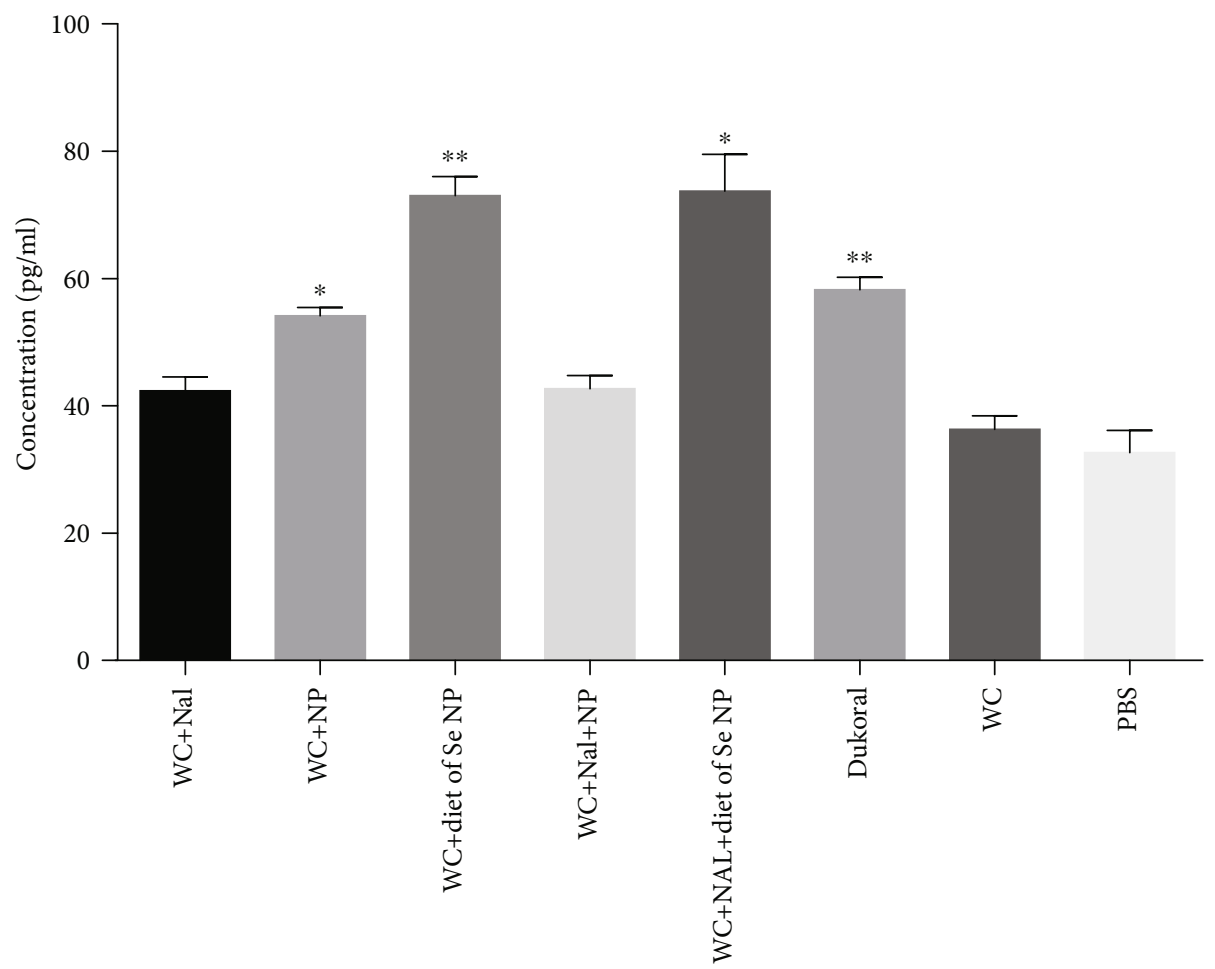

(a)

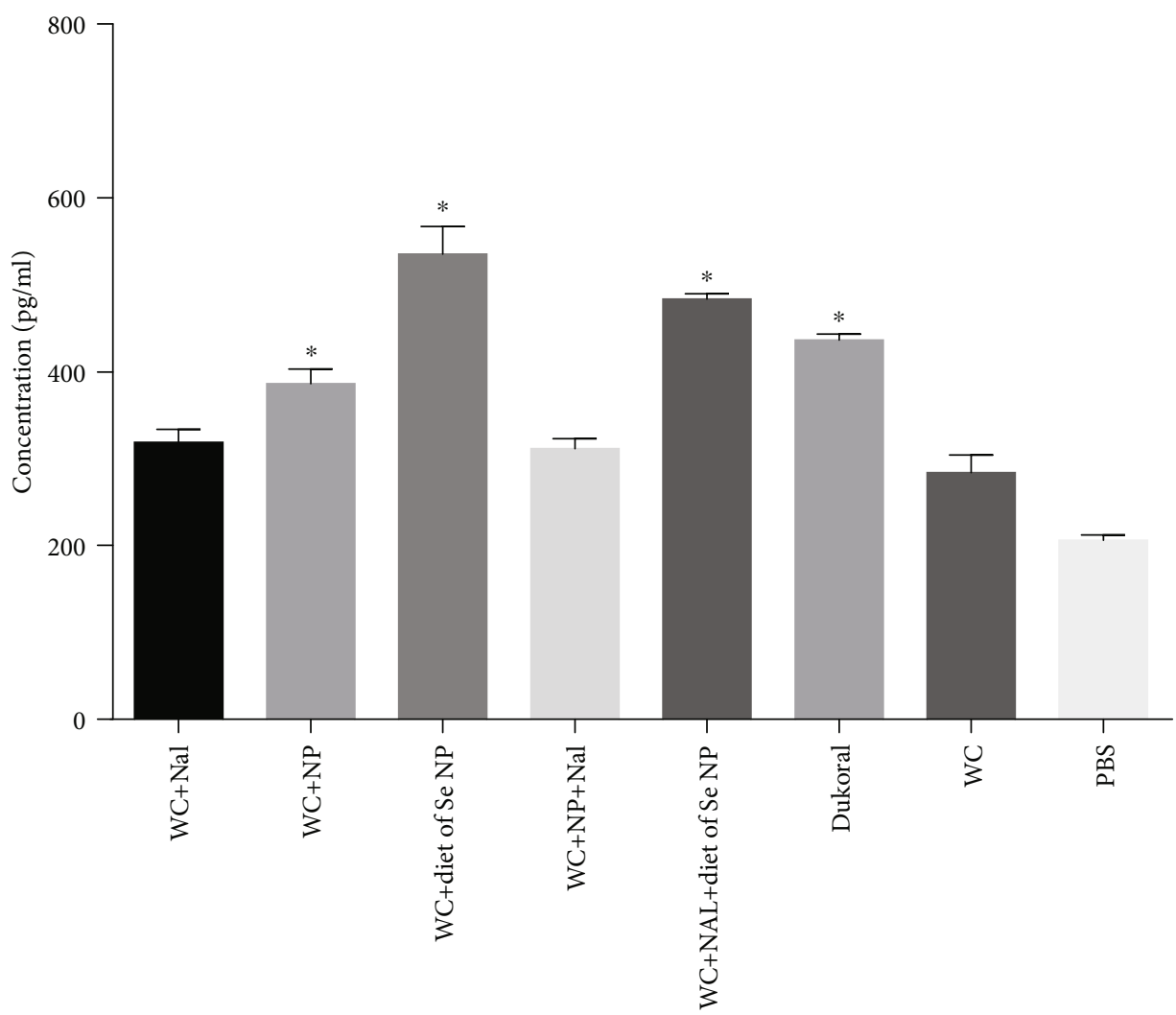

(b)

FIGURE 8: (a) Level of IL-4 in blood on day 42. Immune mouse groups have been compared with the whole-cell group. The data are presented as the mean $\pm \mathrm{SD}\left({ }^{*} P<0.05,{ }^{* *} P<0.01\right)$. (b) Level of IL-5 in blood on day 42 . Immune mouse groups have been compared with the wholecell group. The data are presented as the mean $\pm \mathrm{SD}\left({ }^{*} P<0.05\right)$. 


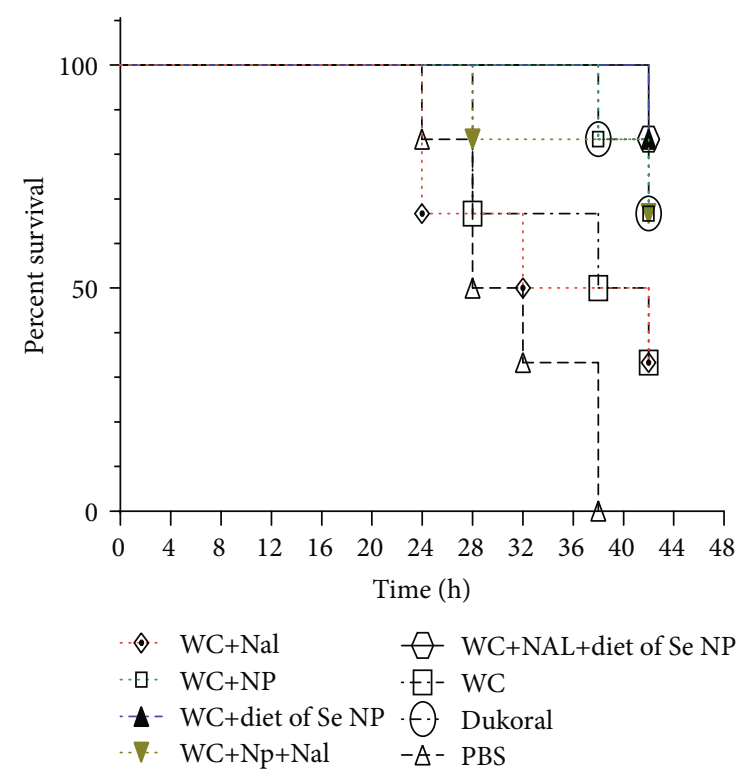

FIGURE 9: Percent survival of neonatal mice in challenge with wholecell $V$. cholerae. Survival curves in neonate challenge were analyzed by the log-rank test $(P<0.001)$.

Previous studies have shown the inhibitory effects of selenium NPs on $V$. cholerae in vitro as well as their inhibitory effect on virulence gene expression of this bacterium [37]. The effect of selenium on the immune system is through molecular mechanisms; however, its precise pathways have not yet been fully established [38]. Actually, selenium plays an important role in immune system function, and its deficiency is associated with impaired innate and adaptive immune responses [38-40]. In a recent study by Mahdavi et al., the effect of selenium NPs as an adjuvant in the mouse model of hepatitis B vaccine was investigated. It was elucidated that selenium NPs were able to increase immune responses against the viruses [41], whereas we substantiate that Se NPs can also be effective as an adjuvant for the vaccine against extracellular bacterial pathogens. Recent studies have demonstrated that selenium nanoparticles in the role of an adjuvant have been able to induce good protective immune responses in the component with vaccine against bacteria and viruses [41-43]. In one study, selenium nanoparticles as an adjuvant in Escherichia coli antigens have been studied and showed that selenium nanoparticle can act as a good adjuvant in immunization [43]. In another research, the immunomodulatory effects of selenium NPs on breast cancer were investigated in a mouse model and it was shown that selenium nanoparticles can induce Th1 and cytokine responses $[44,45]$. Therefore, it can be concluded that selenium nanoparticles as an immune booster can accompany vaccine components to increase the immune responses and to increase their efficacy in the control and prevention of cholera.

Surprisingly, we observed the slightest disparity in immune responses both in antibodies and in cytokines, in mice immunized with naloxone alone and together with Se NPs as an adjuvant for whole cells in comparison with the whole cells alone. Previous studies have shown that naloxone can shift the immune responses to a Th1 pattern and induce robust cellular immune responses against herpes simplex virus type-1 and Listeria monocytogenes (intracellular pathogens) $[13,14]$. However, our results indicated that naloxone as an adjuvant has not been able to adequately increase the immune system against $V$. cholerae and consequently resulted in lower protective immunity.

In conclusion, (i) Se NP can efficiently increase the protective efficacy of $V$. cholerae whole-cell compartment of cholera vaccine, (ii) Se NP as daily diet can more efficiently augment immune responses against $V$. cholerae whole cells, and (iii) naloxone revealed fair function in immunity against cholera.

\section{Data Availability}

The datasets of the current study are available within the article or can be obtained from the corresponding author upon request.

\section{Ethical Approval}

This study protocol was approved by the Ethics Committee of Tarbiat Modares University (code: IR.MODARES.REC.1397.068).

\section{Conflicts of Interest}

The authors declare no conflict of interest.

\section{Acknowledgments}

This study was supported by the research council of Tarbiat Modares University, Tehran, Iran.

\section{References}

[1] S. Mandal, M. D. Mandal, and N. K. Pal, "Cholera: a great global concern," Asian Pacific Journal of Tropical Medicine, vol. 4, no. 7, pp. 573-580, 2011.

[2] Organization WH, “Cholera, 2016," Weekly Epidemiological Record= Relevé épidémiologique hebdomadaire, vol. 92, 2017.

[3] N. Lycke, "Recent progress in mucosal vaccine development: potential and limitations," Nature Reviews Immunology, vol. 12, no. 8, pp. 592-605, 2012.

[4] F. Sallusto, A. Lanzavecchia, K. Araki, and R. Ahmed, "From vaccines to memory and back," Immunity, vol. 33, no. 4, pp. 451-463, 2010.

[5] M. M. Levine, D. R. Nalin, J. P. Craig et al., "Immunity of cholera in man: relative role of antibacterial versus antitoxic immunity," Transactions of the Royal Society of Tropical Medicine and Hygiene, vol. 73, no. 1, pp. 3-9, 1979.

[6] A. A. King, E. L. Ionides, M. Pascual, and M. J. Bouma, "Inapparent infections and cholera dynamics," Nature, vol. 454, no. 7206, pp. 877-880, 2008.

[7] V. A. Feodorova, L. V. Sayapina, M. J. Corbel, and V. L. Motin, "Russian vaccines against especially dangerous bacterial pathogens," Emerging microbes \& infections, vol. 3, no. 1, pp. 1-17, 2019.

[8] A. Dhur, P. Galan, and S. Hercberg, "Relationship between selenium, immunity and resistance against infection," 
Comparative Biochemistry and Physiology Part C: Comparative Pharmacology, vol. 96, no. 2, pp. 271-280, 1990.

[9] H. Zeng and G. F. Combs Jr., "Selenium as an anticancer nutrient: roles in cell proliferation and tumor cell invasion," The Journal of nutritional biochemistry, vol. 19, no. 1, pp. 1-7, 2008.

[10] M. A. Beck, O. A. Levander, and J. Handy, "Selenium deficiency and viral infection," The Journal of nutrition, vol. 133, no. 5, pp. 1463S-1467S, 2003.

[11] H. Tapiero, D. Townsend, and K. Tew, "The antioxidant role of selenium and seleno-compounds," Biomedicine \& pharmacotherapy, vol. 57, no. 3-4, pp. 134-144, 2003.

[12] Z. Huang, A. H. Rose, and P. R. Hoffmann, "The role of selenium in inflammation and immunity: from molecular mechanisms to therapeutic opportunities," Antioxidants \& redox signaling, vol. 16, no. 7, pp. 705-743, 2012.

[13] N. H. Jazani, M. Karimzad, E. Mazloomi et al., "Evaluation of the adjuvant activity of naloxone, an opioid receptor antagonist, in combination with heat-killed Listeria monocytogenes vaccine," Microbes and infection, vol. 12, no. 5, pp. 382-388, 2010.

[14] A. Jamali, M. Mahdavi, Z. M. Hassan et al., "A novel adjuvant, the general opioid antagonist naloxone, elicits a robust cellular immune response for a DNA vaccine," International Immunology, vol. 21, no. 3, pp. 217-225, 2009.

[15] E. Nygren, B.-L. Li, J. Holmgren, and S. R. Attridge, "Establishment of an adult mouse model for direct evaluation of the efficacy of vaccines against Vibrio cholerae," Infection and immunity, vol. 77, no. 8, pp. 3475-3484, 2009.

[16] Z. Zhang, J. Zhang, C. Qu, D. Pan, Z. Chen, and L. Chen, "Label free colorimetric sensing of thiocyanate based on inducing aggregation of Tween 20-stabilized gold nanoparticles," The Analyst, vol. 137, no. 11, pp. 2682-2686, 2012.

[17] T. H. Nguyen, B. Vardhanabhuti, M. Lin, and A. Mustapha, "Antibacterial properties of selenium nanoparticles and their toxicity to Caco-2 cells," Food Control, vol. 77, pp. 17-24, 2017.

[18] A. A. Tarique, A. Kalsy, M. Arifuzzaman et al., "Transcutaneous immunization with a Vibrio cholerae O1 Ogawa synthetic hexasaccharide conjugate following oral whole cell cholera vaccination boosts vibriocidal responses and induces protective immunity in mice," Clinical and Vaccine Immunology, vol. 19, no. 4, pp. 594-602, 2012.

[19] C. IoLARCo and U. L. Animals, Guide for the Care and Use of Laboratory Animals: US Department of Health and Human Services, Public Health Service, National, 1986.

[20] T. I. Crean, M. John, S. B. Calderwood, and E. T. Ryan, “Optimizing the germfree mouse model for in vivo evaluation of oral Vibrio cholerae vaccine and vector strains," Infection and immunity, vol. 68, no. 2, pp. 977-981, 2000.

[21] M. John, E. A. Bridges, A. O. Miller, S. B. Calderwood, and E. T. Ryan, "Comparison of mucosal and systemic humoral immune responses after transcutaneous and oral immunization strategies," Vaccine, vol. 20, no. 21-22, pp. 2720-2726, 2002.

[22] J. E. Rollenhagen, A. Kalsy, R. Saksena et al., "Transcutaneous immunization with a synthetic hexasaccharide-protein conjugate induces anti-Vibrio cholerae lipopolysaccharide responses in mice," Vaccine, vol. 27, no. 36, pp. 4917-4922, 2009.

[23] A. Chernyak, S. Kondo, T. K. Wade et al., "Induction of protective immunity by synthetic Vibrio cholerae hexasaccharide derived from V. cholerae O1 Ogawa lipopolysaccharide bound to a protein carrier," The Journal of infectious diseases, vol. 185, no. 7, pp. 950-962, 2002.

[24] O. mondiale de la Santé and Organization WH, "Cholera vaccines: WHO position paper-August 2017-Vaccins anticholériques: Note de synthèse de l'OMS-août 2017," Weekly Epidemiological Record= Relevé épidémiologique hebdomadaire, vol. 92, no. 34, pp. 477-498, 2017.

[25] Organization WH, "Cholera, 2013," Weekly Epidemiological Record $=$ Relevé épidémiologique hebdomadaire, vol. 89, no. 31, pp. 345-355, 2014.

[26] Organization WH, Oral Cholera Vaccine Stockpile for Cholera Emergency Response, International Coordinating Group on Vaccine Provision for Cholera, Geneva, 2013.

[27] R. E. Begue, G. Castellares, R. Ruiz et al., "Community-based assessment of safety and immunogenicity of the whole cell plus recombinant B subunit (WC/rBS) oral cholera vaccine in Peru," Vaccine, vol. 13, no. 7, pp. 691-694, 1995.

[28] F. P. Van Loon, J. D. Clemens, J. Chakraborty et al., "Field trial of inactivated oral cholera vaccines in Bangladesh: results from 5 years of follow-up," Vaccine, vol. 14, no. 2, pp. 162-166, 1996.

[29] M. Pastor, J. Pedraz, and A. Esquisabel, "The state-of-the-art of approved and under-development cholera vaccines," Vaccine, vol. 31, no. 38, pp. 4069-4078, 2013.

[30] D. Mahalanabis, A. L. Lopez, D. Sur et al., "A randomized, placebo-controlled trial of the bivalent killed, whole-cell, oral cholera vaccine in adults and children in a cholera endemic area in Kolkata, India," PLoS One, vol. 3, no. 6, article e2323, 2008.

[31] J. L. Sanchez, B. Vasquez, R. E. Begue et al., "Protective efficacy of oral whole-cell/recombinant-B-subunit cholera vaccine in Peruvian military recruits," The Lancet, vol. 344, no. 8932, pp. 1273-1276, 1994.

[32] A. M. Khatib, M. Ali, L. Von Seidlein et al., "Effectiveness of an oral cholera vaccine in Zanzibar: findings from a mass vaccination campaign and observational cohort study," The Lancet infectious diseases, vol. 12, no. 11, pp. 837-844, 2012.

[33] F. J. Luquero, L. Grout, I. Ciglenecki et al., "Use of Vibrio cholerae vaccine in an outbreak in Guinea," New England Journal of Medicine, vol. 370, no. 22, pp. 2111-2120, 2014.

[34] Organization WH, The Immunological Basis for Immunization Series: Module 14: Cholera, 2010.

[35] L. A. Dykman, O. A. Volokh, O. V. Gromova et al., "Obtaining and characteristic of antibodies to Vibrio cholerae protective antigens conjugated with gold nanoparticles," in Doklady Biochemistry and Biophysics, Springer, 2020.

[36] M. Fasihi-Ramandi, H. Ghobadi-Ghadikolaee, S. AhmadiRenani, R. A. Taheri, and K. Ahmadi, "Vibrio cholerae lipopolysaccharide loaded chitosan nanoparticle could save life by induction of specific immunoglobulin isotype," Artificial cells, nanomedicine, and biotechnology, vol. 46, no. 1, pp. 5661, 2018.

[37] V. Bhattaram, A. Upadhyay, H.-B. Yin, S. Mooyottu, and K. Venkitanarayanan, "Effect of dietary minerals on virulence attributes of Vibrio cholerae," Frontiers in microbiology, vol. 8, p. 911, 2017.

[38] J. C. Avery and P. R. Hoffmann, "Selenium, selenoproteins, and immunity," Nutrients, vol. 10, no. 9, p. 1203, 2018.

[39] R. Geisberger, C. Kiermayer, C. Hömig et al., "B-and T-cellspecific inactivation of thioredoxin reductase 2 does not 
impair lymphocyte development and maintenance," Biological Chemistry, vol. 388, no. 10, pp. 1083-1090, 2007.

[40] R. K. Shrimali, R. D. Irons, B. A. Carlson et al., "Selenoproteins mediate T cell immunity through an antioxidant mechanism," Journal of Biological Chemistry, vol. 283, no. 29, pp. 2018120185, 2008.

[41] M. Mahdavi, F. Mavandadnejad, M. H. Yazdi et al., "Oral administration of synthetic selenium nanoparticles induced robust Th1 cytokine pattern after HBs antigen vaccination in mouse model," Journal of infection and public health, vol. 10, no. 1, pp. 102-109, 2017.

[42] S. A. Staroverov, A. A. Volkov, S. V. Larionov et al., "Study of transmissible gastroenteritis virus antigen-conjugated immunogenic properties of selenium nanoparticles and gold," Life Science Journal, vol. 11, no. 11, pp. 456-460, 2014.

[43] K. P. Gabalov, M. V. Rumina, T. N. Tarasenko et al., "The adjuvant effect of selenium nanoparticles, Triton X-114 detergent micelles, and lecithin liposomes for Escherichia coli antigens," Applied Biochemistry and Microbiology, vol. 53, no. 5, pp. 587-593, 2017.

[44] M. H. Yazdi, M. Mahdavi, B. Varastehmoradi, M. A. Faramarzi, and A. R. Shahverdi, "The immunostimulatory effect of biogenic selenium nanoparticles on the $4 \mathrm{~T} 1$ breast cancer model: an in vivo study," Biological trace element research, vol. 149, no. 1, pp. 22-28, 2012.

[45] M. H. Yazdi, M. Mahdavi, E. Faghfuri et al., "Th1 immune response induction by biogenic selenium nanoparticles in mice with breast cancer: preliminary vaccine model," Iranian journal of biotechnology, vol. 13, no. 2, pp. 1-9, 2015. 